\title{
Das Prinzip der Winkelstabilität in der Osteosynthese
}

\author{
Lutz Claes
}

\section{Zusammenfassung}

Das Prinzip der winkelstabilen Osteosynthese ist vom Fixateur externe bekannt. Dabei sind die im Knochen verankerten Schrauben so mit einem die Fraktur überbrückenden Kraftträger verbunden, dass sie ihren Einspannwinkel nicht verändern. Winkelstabile Osteosynthesen verzichten auf eine interfragmentäre Kompression und ein Anpressen des Kraftträgers an den Knochen. Die neuen winkelstabilen „Plattenfixateur interne“-Systeme schonen dadurch, wie der Fixateur externe, die periostale Durchblutung, haben jedoch zusätzlich den Vorteil kleinerer interfragmentärer Bewegungen. Diese interfragmentären Bewegungen liegen meistens in einer Größenordnung, die eine Kallusbildung stimuliert und die Knochenheilung begünstigt. Kritische Situationen können für diese Osteosyntheseverfahren bei Schaftfrakturen ohne Abstützung, wegen der hohen Beanspruchung der Implantate, und bei langstreckigen Überbrückungen wegen der erheblichen elastischen Verbiegung der Implantate und der daraus resultierenden hohen interfragmentären Bewegung resultieren. Eine große Bedeutung hat die gute Reposition der Fragmente vor der Osteosynthese, da die steifen Implantate und die winkelstabilen Schrauben keine Korrektur mehr erlauben. Repositionen, die große Frakturspalten zulassen, könnten die Knochenheilung verzögern. Besonders geeignet scheinen winkelstabile Osteosynthesen für Frakturen im metaphysären Knochenbereich zu sein. Dort sind die mechanischen Biegebeanspruchungen für die krafttragenden Implantate geringer als im Schaftbereich, und die winkelstabilen Schrauben erlauben eine gute Verankerung im relativ weichen und manchmal osteoporotischen Knochen.

\section{Einleitung}

Allgemein kann man unter einer winkelstabilen Osteosynthese ein System verstehen, bei dem die im Knochen verankerten Schrauben so mit einem die Fraktur überbrückenden Kraftträger verbunden sind, dass sie sich gegenüber diesem nicht in dem Winkel verschieben können, in dem sie befestigt sind.

Im Unterschied zu den klassischen Osteosyntheseplatten soll dieses Ziel der winkelstabilen Schrauben-Kraftträgerverbindung ohne ein Anpressen des Kraftträgers an den Knochen, d.h. ohne die durch

OP-JOURNAL 2004; 20: 4-7

(c) Georg Thieme Verlag KG Stuttgart · New York die Schraubenzugkräfte erzeugten Haftreibungskräfte erreicht werden.

Solche Osteosynthesesysteme gibt es schon seit langer Zeit. Das klassische Beispiel ist der Fixateur-externe. Auch die subkutane Anwendung in Form des Fixateur interne [4] wird an der Wirbelsäule schon länger praktiziert.

Das Neue ist die Übertragung dieses Befestigungsprinzips auf Implantate für die Frakturversorgung langer Röhrenknochen mit plattenähnlichen extramedullären Systemen. Dies gilt insbesondere für Frakturen im metaphysären Bereich und für Mehrfragmentfrakturen.

Motivation für die Einführung des „Plattenfixateur intern“ war die Vermeidung von Durchblutungsstörungen, wie sie un- ter klassischen Platten mit Kompression zwischen Platte und Knochen auftreten [8] und die Anwendung der minimalinvasiven Chirurgie mit einem Verzicht auf perfekte Reposition und interfragmentäre Kompression [7].

\section{Die Biomechanik der Frakturstabilisierung mit winkelstabilen Implantaten}

Unter biomechanischen Gesichtspunkten handelt es sich bei den winkelstabilen „Plattenfixateur interne“-Osteosynthesen um Verfahren der flexiblen Frakturschienung. Bisher liegen meines Wissens zu diesen neuen Osteosyntheseverfahren nur biomechanische Experimente [5] und noch keine Knochenheilungsstudie vor. Die Kombination von winkelstabilen „Plattenfixateuren interne“ (PC-Fix, Synthes, Chur) [9] und Zugschrauben zur Erzeugung interfragmentärer Kompression war ein Entwicklungsschritt und hat sich nicht durchgesetzt.

Da die biomechanischen Bedingungen jenen beim Fixateur externe weitgehend entsprechen, lassen sich jedoch aus Erfahrungen mit dem Fixateur externe prinzipielle Eigenschaften ableiten.

Bei flexiblen Osteosyntheseformen ist die interfragmentäre Bewegung der entscheidende biomechanische Faktor für den Ablauf der Frakturheilung. Wie bei allen Fixateur-Osteosynthesen werden die interfragmentären Bewegungen überwiegend durch die Verformungen der Schrauben und Kraftträger unter Belastung der stabilisierten Extremität hervorgerufen. Beim Fixateur externe entstehen die größten interfragmentären Bewegungen durch die langen Schrauben, die sich in ihrer freien Länge (L, Abb.1 a) zwischen Knochen und Verankerung am Kraftträger sehr stark durchbiegen. Die Durchbiegung nimmt dabei in der 4.Potenz mit der freien Länge, d.h. überproportional, zu. Interfragmentäre Bewegungen von $1-3 \mathrm{~mm}$ in axialer Richtung des Kno- 
chens sowie auch senkrecht dazu (Scherung in ventro-dorsaler und medio-lateraler Richtung) können bereits bei partieller Belastung der operierten Extremität auftreten [6].

Der große Vorteil der „Plattenfixateure“ liegt darin, dass die freie Länge der Schrauben sehr gering ist, da der Kraftträger nur wenige Millimeter über der Knochenoberfläche zu liegen kommt (Abb.1 b).

Messungen und Berechnungen von Duda [5] mit LISS-Implantaten [7] an Defektosteosynthesen der Tibia haben die interfragmentären Bewegungen und die mechanischen Beanspruchungen des „Plattenfixateur interne“ bestimmt. Unter partieller Belastung von $500 \mathrm{~N}$ axialer Kraft auf das Tibiaplateau ergaben sich bei Defekten, die zwei Schraubenlöcher unbesetzt ließen interfragmentäre Bewegungen, die unter $0,5 \mathrm{~mm}$ lagen. Dabei resultierten die größten Bewegungsanteile aus einer Verbiegung der Platte und einer axialen Stauchung. Scherbewegungen senkrecht zur Knochenlängsachse waren dagegen gering.

Die Biomechanik der winkelstabilen Osteosynthese entspricht jener des Fixateur externe, jedoch sind die interfragmentären Bewegungen bei „Fixateur interne“ Anwendungen geringer.

Bei Fixateur externe Gestaltungen können groß dimensionierte Kraftträger (z.B. großer Durchmesser D) oder mehrere Kraftträger (z.B. AO-Doppelrohrmontage, Abb.1a) eingesetzt werden, was zu einer großen Steifigkeit des Kraftträgers führt, der damit nur einen geringen Anteil an den interfragmentären Bewegungen verursacht. Die Größe der Verformung hängt zusätzlich vom Abstand (A) der beiden frakturnahen Schrauben (A, Abb.1) ab. Je größer dieser Abstand ist, desto größer ist die Verformung.

Bei der Dimensionierung eines „Plattenfixateur interne" müssen zwei Erfordernisse berücksichtigt werden. Auf der einen Seite muss der „Plattenfixateur“ deutlich dicker sein als eine konventionelle Platte, um den biomechanischen Anforderungen zu entsprechen; auf der anderen Seite lassen sich nicht beliebig dicke Implantate unter den Weichteilen platzieren.

Wie die Berechnungen von Duda [5] zeigen, ist die Beanspruchung des Plattenfixateurs bei nicht abgestützten Frakturen

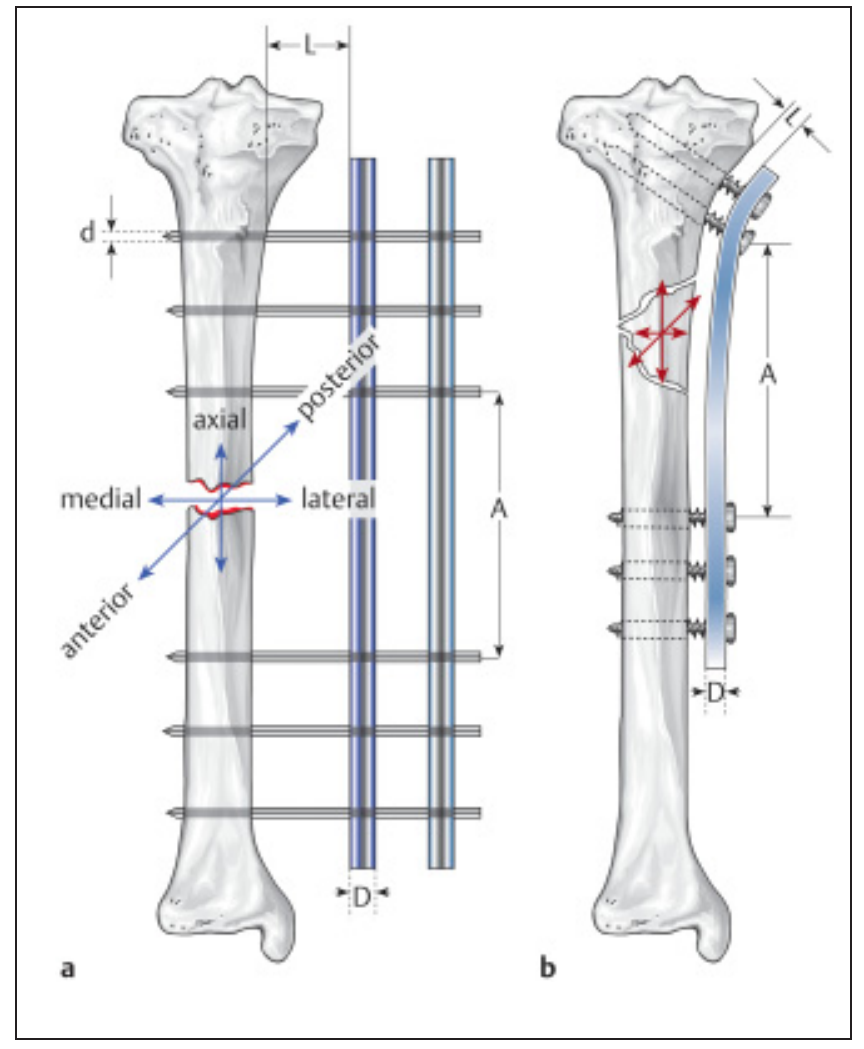

Abb. 1 Schematische Darstellung der wichtigsten mechanischen Faktoren der winkelstabilen Osteosynthese mit Fixateur externe (a) und „Plattenfixateur interne“ (b), die die interfragmentäre Bewegung unter Belastung der Knochen beeinflussen. A: Abstand zwischen den frakturnahen

Schrauben D:

Durchmesser oder Dicke des Kraftträgers L: Freie Länge zwischen Kraftträger und Knochenoberfläche d: Schraubendurchmesser. Die Pfeile stellen die drei Hauptkomponenten der interfragmentären Bewegung dar.

metaphysär unkritisch, kann bei Frakturen im Schaftbereich jedoch kritische Werte im Hinblick auf mechanische Überlastung erreichen. Der begrenzte Querschnitt der „Plattenfixateure“ führt dazu, dass der überwiegende Teil der interfragmentären Bewegung aus ihrer beschränkten Biegesteifigkeit resultiert.

Die mechanische Beanspruchung von „Plattenfixateuren interne“ bei Frakturen im Schaftbereich kann erheblich sein.

Wichtige mechanische Größen sind dabei die Dicke des „Plattenfixateurs interne“ (D) und der Abstand (A) zwischen den beiden frakturnahesten Schrauben in den Hauptfragmenten (Abb.1 b). Je kleiner die Dicke (D) des „Plattenfixateurs interne" und je größer der Abstand (A) ist, desto größer ist die unter Belastung resultierende interfragmentäre Bewegung.

Für den Bereich zwischen den beiden frakturnahen Schrauben gelten im Prinzip die gleichen Bedingungen, wie sie für die überbrückenden Plattenosteosynthesen mit konventionellen Platten gelten. Liegen in diesen Bereichen mehrere Fragmente und Frakturspalten vor, so kann sich die interfragmentäre Bewegung zwischen dem distalen und proximalen Hauptfragment auf mehrere Frak- turspalten verteilen und damit lokal kleiner werden.

Die kritische interfragmentäre Bewegung resultiert beim Fixateur externe aus der freien Schraubenlänge, beim „Plattenfixateur interne“ aus der geringen Plattendicke und dem eventuell großen Abstand zwischen den Schrauben der Hauptfragmente.

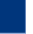
Bei überbrückenden Osteosynthesen mit großen Abständen zwischen den Hauptfragmenten kann es zu erheblichen elastischen Verformungen der Osteosynthese kommen.

Die aus Festigkeitsanforderungen erforderliche große Plattendicke und die daraus resultierende fehlende Anpassungsmöglichkeit an die individuellen anatomischen Bedingungen sowie die winkelstabile Schraubenfixation schränken die Repositionsqualität der Fragmente ein. Dies kann zu relativ großen Frakturspalten führen, die eine Ursache für Heilungsverzögerungen sein können.

Ein Vorteil der winkelstabilen Implantate gegenüber den konventionellen Plattenosteosynthesen wird bei der Stabilisierung von Frakturen des osteoporotischen Knochens diskutiert. 
Dabei muss man jedoch zwei Probleme unterscheiden, die aus der biomechanischen Schwäche des osteoporotischen Knochens für die Primärstabilität der Osteosynthese resultieren. Das erste Problem bei der klassischen Plattenosteosynthese ist das Anziehen der Plattenschrauben, um eine ausreichende Kompression zwischen Platte und Knochen für eine stabile Osteosynthese zu erreichen. Hier kann es bei osteoporotischen Knochen bereits zum „Durchdrehen“ der Schrauben, d.h. zu einer irreversiblen Verformung des Knochens um die Schrauben kommen. Dies hat zur Folge, dass die erzielten Schraubenzugkräfte relativ niedrig sind und die Primärstabilität beeinträchtigt ist. Diese Komplikationsmöglichkeit ist beim „Plattenfixateur intern" mit seinen winkelstabilen Schrauben ausgeschlossen, da der Plattenfixateur nicht mit Schraubenzugkräften gegen den Knochen fixiert wird.

Ein zweites Problem bei der Verankerung von Schrauben im osteoporotischen Knochen ist dadurch jedoch nicht gelöst. Es ist das Problem, dass Schrauben, die im Knochen verankert sind, nicht nur auf Zug belastet sein können, sondern auch z.B. Biegemomente und Scherkräfte aufnehmen müssen. Die Beanspruchungen an der Grenzfläche zum Knochen hängen dabei von der äußeren Belastung der Schraube, dem Schraubendurchmesser und der Schraubenlänge im Knochen ab. Sind die Beanspruchungen höher als die Festigkeit des osteoporotischen Knochens, so kommt es zur irreversiblen Verformung des Knochens und damit $\mathrm{zu}$ einem Durchschneiden der Schraube durch den Knochen (cut out). Diese Komplikation kann auch bei einer ohne Schraubenvorspannung eingesetzten winkelstabilen Schraube auftreten.

Während bei einer konventionellen Platte die Beanspruchungen durch die Schraubenzugkräfte dominieren, sind beim Fixateur externe die Biegemomente kritisch die durch die Schrauben auf den Knochen übertragen werden und bei den winkelstabilen „Plattenfixateur externe“ sind die Scherkräfte die kritische Beanspruchung.

\section{Die Knochenheilung mit winkelstabilen Osteosynthesen}

Die Knochenheilung mit winkelstabilen „Plattenfixateuren interne“ und einem Verzicht auf interfragmentäre Kompression wurde bisher noch nicht studiert. Sie dürfte sich jedoch nicht wesentlich von jener unter Fixateur-externe-Stabilisierung unterscheiden. Im Allgemeinen wird es sich um die Knochenheilung mit Frakturspalten und relativ geringen interfragmentären Bewegungen handeln, da bei winkelstabilen Implantaten auf eine interfragmentäre Kompression verzichtet wird. Bei Frakturspalten bis zu $2 \mathrm{~mm}$ führten bereits sehr kleine interfragmentäre Bewegungen unter 0,1 mm zur Knochenneubildung in Form eines kleinen Kallus und einer Knochenneubildung im Frakturspalt [3] (Abb. 2). Solche Bedingungen sind in der Frakturzone auf der plattennahen Seite zu erwarten. Größere interfragmentäre Bewegungen bis zu 0,5 mm, wie sie beim LISS-System gemessen wurden [5], stimulierten die Kallusbildung stärker und führen vor allem auf der, dem „Plattenfixateur“ gegenüber liegenden Seite zur Kallusüberbrückung [1] (Abb. 3).

Die kleinen interfragmentären Bewegungen unter Belastung der winkelstabilen internen Plattenfixateure stimulieren eine Kallusheilung.

Noch größere interfragmentäre Bewegungen können zu einer Resorption der Fragmentenden und zu einer Vergrößerung der Frakturspalten führen. Solche Bedingungen wie auch große Frakturspalten können die Frakturheilung verzögern [1]. Dabei gilt, dass bei Frakturspalten, die größer als $2 \mathrm{~mm}$ sind, geringe interfragmentäre Bewegungen zur schnelleren Heilung führen als instabile Situationen. Dies scheint insbesondere für die „Plattenfixateur interne“ -Anwendung von Bedeutung zu sein, da hier aufgrund der fehlenden Möglichkeit der Plattenanpassung an die anatomische Situation und der vorgegebenen Richtung der Schraubenlöcher eine ideale Reposition häufig nicht möglich ist und relativ große Frakturspalten entstehen können [7]. Unter ungünstigen Bedingungen, d. h. zum Beispiel bei großen Frakturspalten und starken Verformungen des „Plattenfixateur interne“ aufgrund der beschränkten Biegesteifigkeit und großer Abstände zwischen den fragmentnahen Schrauben, könnten deshalb ungünstige Heilungsbedingungen entstehen. Eine solche Situation könnte z. B. bei der langstreckigen Überbrückung eines großen Fragmentes auftreten, wo eine erhebliche elastische Plattenverformung und die daraus resultierende große interfragmentäre Bewegung auf nur zwei Frakturspalten verteilt wird. Bei Mehrfragmentfrakturen verteilen sich diese Bewegungen auf mehrere Frakturspalten und

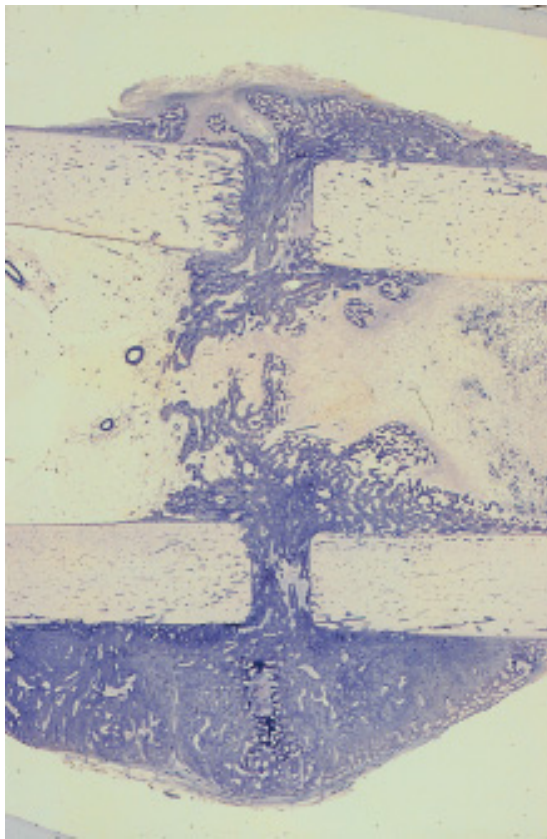

Abb. 2 Längsschnitt durch einen Schafsmetatarsus mit einem Frakturheilungskallus 9 Wochen p. o. (Färbung nach Paragon). Die Modellfraktur (Osteotomie) mit einer stabilen Fixation (interfragmentäre Bewegung ca. $0,1 \mathrm{~mm}$ ) ist mit einem peripheren Kallus überbrückt. Der kortikale Osteotomiespalt beginnt sich mit Geflechtknochen aufzufüllen.

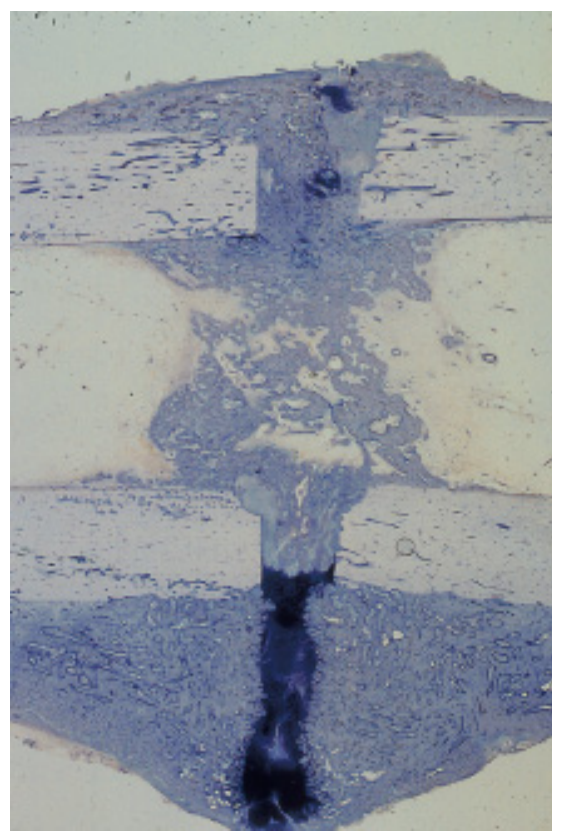

Abb. 3 Längsschnitt durch einen Schafsmetatarsus mit einem Frakturheilungskallus 9 Wochen p. o. (Färbung nach Paragon). Die Modellfraktur (Osteotomie) wurde flexibel stabilisiert (interfragmentäre Bewegung ca. $0,5 \mathrm{~mm}$ ). Die größere interfragmentäre Bewegung stimuliert eine größere Kallusbildung vor allem auf der dem Fixateur abgewandten Seite und eine stärker ausgeprägte enchondrale Ossifikation. 


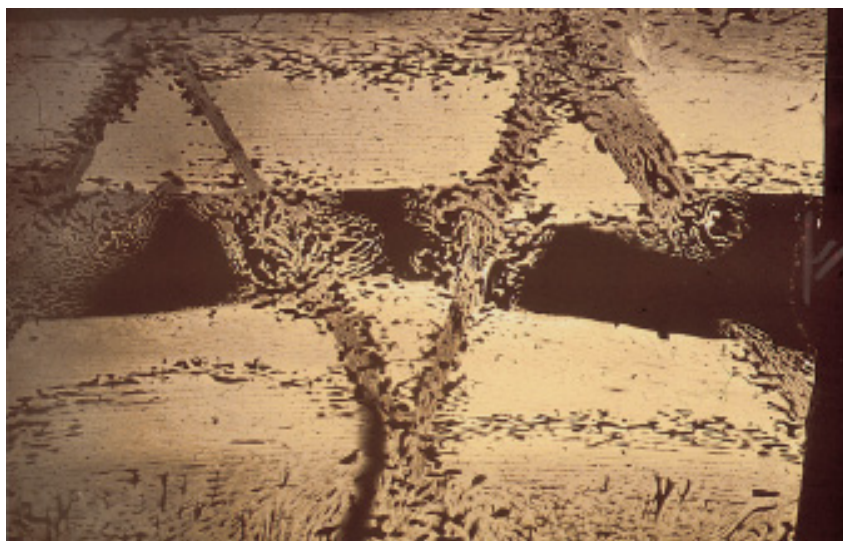

Abb.4 Mikroradiografie einer Mehrfragmentmodellfraktur mit drei freien Fragmenten. Die freien Fragmente wurden nicht stabilisiert. Ein überbrückender Fixateur externe stabilisierte die beiden Hauptfragmente. Zwölf Wochen p. o. waren die Fragmente mit Kallus überbrückt und die Osteotomiespalten mit Geflechtknochen aufgefüllt.

sind damit besser tolerierbar für die Knochenneubildung. Ein experimentelles Beispiel für eine solche Situation ist die Stabilisierung einer Modellfraktur mit drei freien Fragmenten, die sowohl mit einem steifen Fixateur externe als auch mit einer Überbrückungsplatte gut ausgeheilt sind [2]. Die Fragmente zeigen im tierexperimentellen Modell am Schaf nach 12 Wochen eine Kallusüberbrükkung der Fragmente und eine Auffüllung der Frakturspalten mit Geflechtknochen (Abb.4). Wie beim „Plattenfixateur interne" haben diese beiden Stabilisationsverfahren gegenüber der konventionellen Technik mit stabiler Fixation aller Fragmente, durch interfragmentäre Kompression und Plattenanpressung, den Vorteil der geringeren biologischen Beeinträchtigung des Heilungsgebietes.

Überbrückende winkelstabile Osteosynthesen schonen bei Mehrfragmentfrakturen die lokale Vaskularität und begünstigen die Knochenheilung.

Alle diese Untersuchungen wurden an diaphysären Frakturen und Frakturmodellen und nicht im Bereich der metaphysären Frakturen durchgeführt. Hier gibt es bisher kaum experimentelle Untersuchungen zur Knochenheilung. Klinische Erfahrungen und die Untersuchungen zur stabilen Spaltheilung [10] deuten jedoch darauf hin, dass im spongiösen Knochenbereich die Heilung schneller und unkritischer abläuft als im kortikalen Knochen.

\section{Literatur}

${ }^{1}$ Claes L, Augat P, Suger G et al. Influence of size and stability of the osteotomy gap on the success of fracture healing. J Orthop Res 1997; 577-584

${ }^{2}$ Claes L, Heitemeyer U, Krischak G et al. Fixation technique influences osteogenesis of comminuted fractures. Clin Orthop 1999; $221-229$.

${ }^{3}$ Claes LE, Wilke HJ, Augat P et al. Effect of dynamization on gap healing of diaphyseal fractures under external fixation. Clin Biomech 1995; 227-234

${ }^{4}$ Dick W. The „fixateur interne“ as a versatile implant for spine surgery. Spine 1987; 882 900

${ }^{5}$ Duda GN, Mandruzzato F, Heller M et al. Mechanical conditions in the internal stabilization of proximal tibial defects. Clin Biomech 2002; 64-72

${ }^{6}$ Duda GN, Sporrer S, Sollmann M et al. Interfragmentary movements in the early phase of healing in distraction and correction osteotomies stabilized with ring fixators. Langenbecks Arch Surg 2003; 433-440

${ }^{7}$ Haas NP, Schütz M, Hoffmann R et al. LISS Less Invasive Stabilization System - ein neuer Fixateur intern für distale Femurfrakturen. OP-Journal 1997; 340 - 344

8 Perren SM, Buchanan JS. Basic concepts relevant to the design and development of the Point Contact Fixator (PC-Fix). Injury 1995; S-B1-4

${ }^{9}$ Tepic S, Remiger AR, Morikawa $\mathrm{K}$ et al. Strength recovery in fractured sheep tibia treated with a plate or an internal fixator: an experimental study with a two-year follow-up. J Orthop Trauma 1997; 14-23

${ }^{10}$ Uhthoff HK, Rahn BA. Healing patterns of metaphyseal fractures. Clin Orthop 1981; $295-303$ Bewegungen erlauben. Eine gute Reposition der Fragmente vor der Osteosynthese erscheint besonders wichtig. Repositionsfehler können wegen der steifen Implantate und der definierten Lage der winkelstabilen Schrauben nicht korrigiert werden. Daraus resultierende große Frakturspalten könnten die Frakturheilung verzögern.

Das Prinzip der winkelstabilen Osteosynthese scheint besonders für die metaphysären Frakturen geeignet zu sein. Die Biegebeanspruchungen der „Plattenfixateur interne" sind im gelenknahen Bereich unkritischer und die Vorteile der winkelstabilen Schraubenverankerung kommen im spongiösen Knochen mehr zum Tragen als im kortikalen Bereich des diaphysären Knochens.

\section{Prof. Dr. med. Lutz Claes}

Direktor

Institut für Unfallchirurgische Forschung und Biomechanik Universität Ulm Helmholtzstraße 14 D-89081 Ulm 